\title{
ERWARTUNGEN
}

\section{Ein Gewinn für alle}

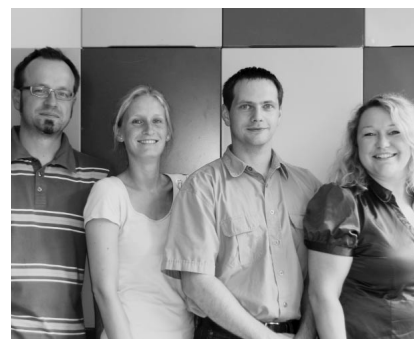

VON DORIS BUCHHOLZER, HANNA NEZADAL, CHRISTOPH STREUBEL, MARTIN WALZ Die Autoren dieses Beitrags haben sich nach einem Diplomstudium der Sozialpädagogik und nach Berufstätigkeit für den Erwerb einer zusätzlichen Qualifikation als »Master of Social Management « entschlossen (auf dem Foto von links nach rechts): Christoph Streubel hat langjährige Erfahrung in der Suchtund Jugendhilfe sowie als Betriebsrat, derzeit ist er im Bereich ambulanter Erziehungshilfen tätig.

Hanna Nezadal hat Berufserfahrung in der Medienpädagogik und der Erwachsenenbildung; derzeit ist sie in der Jugendberufshilfe tätig. Martin Walz sammelte langjährig Erfahrungen in der Kinder- und Jungendhilfe; derzeit ist er Leiter einer Kindertagesstätte. Doris Buchholzer hat Berufserfahrung in der gesetzlichen Betreuung und in der Arbeit mit erwachsenen Menschen mit Autismus; derzeit ist sie in der Aids-Hilfe tätig.

E-Mail doris.buchholzer@web.de
Viele Fachkräfte der Sozialen Arbeit wollen sich durch ein zusätzliches Masterstudium für Führungsaufgaben qualifizieren. Die Erfahrungen, die sie bei der Ausbildung und bei der Hoffnung auf einen Karriereschritt machen, sind dabei durchaus gemischt.

Angestoßen durch den Bologna-Prozess von 1999 soll bis 2010 ein gemeinsamer Hochschulraum in Europa errichtet werden. Im Hinblick auf das zehnjährige Jubiläum und dessen Zielvorgaben ist die Hochschulreform zwar mittlerweile weit fortgeschritten, wird bis zum Jahrende jedoch nicht vollendet sein. Im Wintersemester 2008/09 entsprachen 75 Prozent des gesamten Studienangebots an deutschen Hochschulen den zweigliedrigen Bachelor- und Masterstudiengängen.

Auch bei den Studiengängen der Sozialen Arbeit hat die Reform zu weitreichenden Veränderungen geführt. In der Praxis zeigt sich bereits, dass die Bachelor-Abschlüsse in diesem Bereich den Diplom-Abschlüssen Sozialarbeiter/Sozialpädagoge* gleichgestellt werden. Folglich und durch die gegebene Umstrukturierung finden Aufbau- und berufsbegleitende Weiterbildungsmaster im sozialen Bereich einen immer höheren Zuspruch.

Weit verbreitet ist inzwischen der »Master of Social Management (M.S.M.) «. So entsprachen 2007 von etwa 120 angebotenen Master (MA)-Studiengängen für Soziale Arbeit etwa 30 dem Sozialmanagement. Auf der Grundlage dieser Entwicklungen und der damit verbundenen Unsicherheit bezüglich der zukünftigen Wertigkeit des Diplomabschlusses entschieden sich die vier Autoren - allesamt Diplom-Sozialpädagogen $(\mathrm{FH})$-, einen international anerkannten Masterabschluss zu erwerben.

Die Fachrichtung Sozialmanagement ergab sich aus dem Wunsch, sich für leitende und koordinierende Aufgaben im sozialen Arbeitsfeld weiterzuqualifizieren und somit bessere Aufstiegschancen zu erwerben. Aus dem noch unübersichtlichen Dschungel der Angebote fiel die Entscheidung auf das Angebot in Nürnberg aus einer Kombination folgender Gründe: berufsbegleitender Präsenzstudiengang (mit Vorteilen gegenüber dem Fernstudium, etwa die persönliche Betreuungs- und Rücksprachemöglichkeiten), ansprechendes Preis-Leistungsverhältnis, Wohnortnähe, Ausgewogenheit fachlicher und humanistischer Inhalte, persönliche Empfehlungen.
Kurz vor Beendigung des Studiums stellt sich die - aus Ermangelung an Erfahrungsberichten der vorhergehenden Absolventen eher spekulative Frage, wie ein mögliches Szenario aussehen könnte, wenn ein Masterabsolvent des Sozialmanagement in einer Leitungsposition sein Wissen anwendet.

\section{Masterabsolventen treten in Aktion - mögliche Szenarien}

Die Verbesserung der Professionalität und Struktur sollten einem M.S.M.-Absolventen zunächst besonders am Herzen liegen. Er setzt sofort seine neu erworbenen Fähigkeiten ein, die er zum Beispiel in den Gebieten Mitarbeiterführung, Finanzierung oder Controlling erlernt und erprobt hat. Er führt dementsprechend Zielvereinbarungs-, Kritik- und Rück- meldegespräche mit den Mitarbeitern seiner Einrichtung. $\mathrm{Zu}$ seinem Erstaunen stößt er auf Misstrauen, Widerstand oder gar Schweigen. Er ist enttäuscht. Schließlich ging es im Miteinander bisher ja auch ganz gut ohne solche Chefmaßnahmen und rein auf Vertrauensbasis.

Selbstverständlich hat unser Absolvent auch bezüglich der Finanzierung und Effizienz des Unternehmens Verbesserungsvorschläge und Umgestaltungswünsche. Zu seiner Überraschung werden diese Anregungen ebenfalls nicht beachtet, abgeschmettert oder es gibt ganz wichtige Gründe, warum dies gerade in dieser Einrichtung, in diesem Unternehmen nicht durchführbar ist.

\section{Was ist in diesem Szenario schief gelaufen?}

Dass Systeme zu einer beachtlichen Trägheit neigen und Veränderungen gegenüber einen kräftezehrenden Widerstand aufbauen können, wissen wir aus der Systemtheorie und in der Praxis spätestens seit der Einführung der Personalcomputer in sozialen Unternehmen. Der Masterabsolventen kann nichts weiter tun, als die Ärmel hochzukrempeln, sich auf starken Gegenwind einzustellen und einen Gang zurückzuschalten und somit Veränderungen Schritt für Schritt einzuführen. Um nicht zu resignieren oder ein negatives Menschenbild zu entwickeln, muss sich der Absolvent vor Augen führen, dass er von dem kom- 
plexen Beziehungsgeflecht einer Einrichtung auf keinen Fall eine schnellere Auseinandersetzung mit neuen Strukturen erwarten kann, als er sie selbst hatte. Und das sind immerhin mindestens die zweieinhalb Jahre der Ausbildung.

\section{Sind Absolventen des Master- studiengangs in der beruflichen Praxis für alle Ebenen sozialer Organisationen ein Gewinn?}

Diesen Widerständen zum Trotz sind Absolventen eines berufsbegleitenden Masterstudiengangs für alle Ebenen im Sozialbereich gewinnbringend. Die Zulassung zum Studium erfordert zwei Jahre Berufserfahrung, doch ein Großteil der Studierenden kann auf weit mehr Praxiserfahrung zurückblicken. So haben die Basismitarbeiter in den Einrichtungen den Vorteil, dass die zukünftigen Führungskräfte praxiserfahrene Sozialpädagogen sind, die die Möglichkeiten und Schwierigkeiten in der alltäglichen Arbeit aus der eigenen Vergangenheit kennen, diese in ihre Überlegen bereits im Vorfeld einbeziehen und kritische Anmerkungen der Mitarbeiter einordnen und nachvollziehen können.

Als zukünftige Leitungskräfte sehen sie auch, welche Anforderungen an die Bereichsleitungen auf ihren "Sandwichpositionen " gestellt werden. Als Schnittstelle zwischen Basis und Geschäftsführung müssen sie unterschiedlichen Erwartungen gerecht werden, sind Informationsvermittler und müssen kritische Leitungsentscheidungen mittragen und kommunizieren. Um erfolgreich zu sein, benötigen Bereichsleitungen den Rückhalt der Geschäftsführung und einen klar beschriebenen Kompetenzbereich, innerhalb dessen sie frei entscheiden können.

$\mathrm{Zu}$ guter Letzt sind Führungskräfte mit Sozialmanagementausbildung auch für die Träger der Einrichtung und alle relevanten Stakeholder von Nutzen. Sozialmanager kennen die komplexen Zusammenhänge von Führung und haben Kenntnisse in allen wichtigen Fachbereichen, um die Einrichtung im sozialen und gesamtgesellschaftlichen Kontext zu sehen. So können Sozialmanager zielorientiert steuern und auch das langfristige Bestehen des Sozialunternehmens sicherstellen.

\section{Anmerkung}

* Aus Gründen der besseren Lesbarkeit wird die grammatische Form verwendet, welche die weibliche selbstverständlich mit einschließt.

\section{Literatur}

Nodes, Wilfried (Hg.) (2007), Masterstudiengänge für die Soziale Arbeit, München. Internet http://www.agj.de/pdf/5/BA_MA_ Studiengaenge.pdf [4.6.2009]

\section{Können sich die Absolventen des Masterstudienganges Sozial- management in der sozialen Landschaft als kritische Denker mit einem klaren Ziel positionieren?}

Die Aufgabe des Sozialmanagements wurde in der Vergangenheit von Praktikern ohne Managementkenntnisse oder durch Betriebswirte ohne Bezug zum Sozialbereich wahrgenommen. Aufgrund der knapper werdenden finanziellen Mittel wird in allen Bereichen der Sozialen Arbeit unternehmerisches Denken immer wichtiger. Da die Absolventen des Masterstudienganges Sozialmanagement sowohl soziale als auch wirtschaftliche Aspekte im Blick haben, erfüllen sie gute Voraussetzungen, sich als zukünftige Manager in der sozialen Landschaft zu positionieren.

Sozialmanager verfolgen das Ziel des Fortbestehens der sozialen Einrichtung und nicht der Profitgenerierung per se. Sie sind die »neue Generation «von Sozialpädagogen, die Qualität und Effizienz propagieren und dabei von einer moralischen Verpflichtung geleitet werden (sollten).

Managementinstrumente können und müssen von ihnen gezielt und verantwortungsbewusst ausgewählt, und an die Bedürfnisse der sozialen Einrichtung angepasst werden. Da Sozialmanager neben Kenntnissen der Managementfunktionen und Besonderheiten des Sozialbereichs über ein hohes Maß an sozialen Kompetenzen verfügen, können sie unternehmerische Aspekte in die soziale Landschaft und das Handlungsbewusstsein derer Akteure integrieren. Somit können schließlich Professionalität und Innovation vorangetrieben werden. Die Absolventen des Masterstudienganges Sozialmanagement werden jedoch vor der Herausforderung stehen, die Verantwortlichen der Wohlfahrtsverbände und sozialen Organisationen von ihren umfassenden Fähigkeiten zu überzeugen und sich zu etablieren.

\section{Wie sieht es mit der grundsätzli- chen und finanziellen Würdigung des Abschlusses M.S.M. aus?}

Dazu müssen wir zunächst wieder den Bogen zum Bologna-Prozess einerseits und dem Tarifvertrag des öffentlichen Dienstes (TVöD) andererseits, der »Leitwährung « für die Soziale Arbeit, schlagen.

Nach dem TVöD sollen Fachhochschulabschlüsse in die Entgeltgruppen (EG) 9 bis 12 eingestuft werden. So werden zurzeit die Diplom-Sozialpädagogen/Sozialarbeiter und Bachelor Soziale Arbeit vorläufig in die EG 9 eingruppiert (Stufe $1=2.237,38$ Euro, Stufe 6 = 3.423,37 Euro). Hochschul- und MasterAbschlüsse sollen in die EG 13 bis $15 \ddot{U}$ eingruppiert werden (z. B. EG 13, Stufe 1 = 3.038,64 Euro, Stufe $6=4.589,23$ Euro).

Zum 1. November 2009 traten die neu vereinbarte Regelungen zur Eingruppierung der Beschäftigten im Sozial- und Erziehungsdienst in Kraft. Sie gelten vorläufig bis zum 31. Dezember 2014. Die Tätigkeitsmerkmale der Anlage 1 a BAT (Bundesangestelltentarif) für die »Angestellten im Sozial- und Erziehungsdienst " bleiben unverändert bestehen. Sie werden jedoch nunmehr Bestandteil eigenständiger Eingruppierungsregelungen und einer eigenen Entgelttabelle. Nach dieser Entgelttabelle (Anlage C TVöD - Tarifgebiet West) bewegt sich das Gehalt von Sozialpädagogen/Sozialarbeitern zwischen der neuen Entgeltgruppe S 11, Stufe $1=2.300,00 \mathrm{Eu}-$ ro und der S 18, Stufe $6=4.525,00$ Euro. In Bezug zum TVöD wird die höchste Entgeltgruppe S 18 der EG 12 gleichgesetzt. Folglich umfasst die neue Eingruppierungsregelung nicht die Master-Abschlüsse bei den Studiengängen der Sozialen Arbeit. Zudem ergab eine aktuelle Studie der Arbeitsgemeinschaft für Kinder- und Jugendhilfe (AGJ), dass zur Berufseinmündung von $\mathrm{Ba}$ chelor- und Master-Absolventen im Bereich Soziale Arbeit bislang noch keine breiten Erfahrungen oder empirischen Auswertungen vorliegen. Dieselbe Studie stellt jedoch auch fest, dass für Absolventen von Masterstudiengängen in der Regel Leitungsaufgaben oder Aufgaben von wissenschaftlichen Mitarbeitern vorgesehen sind. In der Konsequenz also ein mehr an Arbeit und Verantwortung für das nahezu gleiche Geld?

Inzwischen gibt es im sozialen Sektor die Möglichkeit, durch Verhandlungsgeschick eine adäquate Bezahlung zu erzielen. Wir befinden uns jedoch weiterhin im Non-Profit-Bereich. Soziale Kostenträger sind naturgegeben zurückhaltend und orientieren sich bestenfalls an einer Leitwährung, die der TVöD für die soziale Arbeit eben darstellt.

Natürlich kann die Übernahme von Verantwortung schon Reiz genug sein, sich auf diese Aufgabe mit dem M.S.M.-Studium vorzubereiten oder zu verbessern. Das ist tatsächlich auch ein wesentlicher Grund der bisherigen Absolventen, sich für dieses Studium zu entscheiden. Allerdings gehen die Übernahme von Verantwortung und deren entsprechende finanzielle Würdigung zurzeit (noch) nicht Hand in Hand.

Neben der Eingruppierungs- und Entlohnungsdebatte ist also auch die Professionalisierungsdebatte in der Sozialen Arbeit eine Konstante. Sie wird nicht nur innerhalb, sondern auch außerhalb ihrer Profession geführt. Der M.S.M. kann einen wesentlichen Beitrag dazu leisten. Auf Dauer wird sich der Abschluss " Master of Social Management « jedoch nur etablieren, wenn damit eine berufliche und somit auch finanzielle Perspektive verbunden ist. 